\title{
Kinematics of the Spine During Sit-to-Stand Movement Using Motion Analysis Systems: A Systematic Review of Literature
}

\author{
Mohammad Reza Pourahmadi, Ismail Ebrahimi Takamjani, Shapour Jaberzadeh, \\ Javad Sarrafzadeh, Mohammad Ali Sanjari, Rasool Bagheri, and Morteza Taghipour
}

\begin{abstract}
Context: Clinical evaluation of the spine is commonplace in musculoskeletal therapies, such as physiotherapy, physical medicine/rehabilitation, osteopathic, and chiropractic clinics. Sit-to-stand (STS) is one of the most mechanically demanding daily activities and crucial to independence. Difficulty or inability to perform STS is common in individuals with a variety of motor disabilities, such as low back pain (LBP). Objective: The purpose of this systematic review was to evaluate available evidence in literature to determine 2-dimensional and 3-dimensional kinematics of the spine during STS in patients with LBP and healthy young adult participants using motion analysis systems (electromagnetic and marker based). Methods: Electronic databases (PubMed/MEDLINE [National Library of Medicine], Scopus, ScienceDirect, and Google Scholar) were searched between January 2002 and February 2017. Additionally, the reference lists of the articles that met the inclusion criteria were also searched. Prospective studies published in peer-reviewed journals, with full text available in English, investigating the kinematics of the spine during STS in healthy subjects (mean age between 18 and $50 \mathrm{y}$ ) or in patients with LBP using motion analysis systems, were included. Sixteen studies fulfilled the eligibility criteria. All information relating to methodology and kinematic modeling of the spine segments along with the outcome measures was extracted from the studies identified for synthesis. Results: The results indicated that the kinematics of the spine are greatly changed in patients with LBP. In order to develop a better understanding of spine kinematics, studies recommended that the trunk should be analyzed as a multisegment. It has been shown that there is no difference between the kinematics of patients with LBP and healthy population when the spine is analyzed as a single segment. Furthermore, between-gender differences are present during STS movement. Conclusion: This review provided a valuable summary of the research to date examining the kinematics of the spine during STS.
\end{abstract}

Keywords: low back pain, functional activity, biomechanical phenomena, vertebral column

Sit-to-stand (STS) movement and its reverse, which are considered fundamental prerequisites for daily activities and functional independence, are repeated many times throughout the day. ${ }^{1,2}$ Hughes et $\mathrm{al}^{3}$ reported that STS is the most frequently performed functional activity in daily life. This maneuver is quite demanding from a neuromuscular perspective and is often affected by pathology and age..$^{1,4-8}$ STS consists of transferring the center of mass from a low position centered within a base of support to a high position over a shallow base of support. ${ }^{9}$ In addition, STS movement requires around $60 \%$ of total sagittal-plane lumbar mobility per day..$^{10,11}$ It has been shown that people who have difficulty rising to a standing position have a greater likelihood of falling during ambulation ${ }^{3,12}$ and need help with daily activities. ${ }^{13,14}$ Inability to stand up has been linked to death in elderly people. ${ }^{15}$ As a result, studying STS is encouraged by the fact that this maneuver is frequently described as painful by patients with chronic low back pain (CLBP) and is often addressed in rehabilitation programs. ${ }^{16}$

Normal spinal mobility is required for optimal performance of daily activities, and it has been reported that the impairment of

Pourahmadi, Ebrahimi Takamjani, Sarrafzadeh, and Bagheri are with the Department of Physiotherapy, School of Rehabilitation Sciences, Iran University of Medical Sciences, Tehran, Iran. Jaberzadeh is with the Faculty of Medicine, Department of Physiotherapy, School of Primary Healthcare, Nursing and Health Sciences, Monash University, Melbourne, Australia. Sanjari is with the Department of Rehabilitation Basic Sciences, School of Rehabilitation Sciences, Iran University of Medical Sciences, Tehran, Iran. Ebrahimi Takamjani is with the Department of Physiotherapy, University of Social Welfare and Rehabilitation Sciences, Tehran, Iran. Ebrahimi Takamjani (dr_i_ebrahimi@yahoo.com) is corresponding author. spinal mobility can result in various forms of functional disabilities, ${ }^{17}$ which may have serious adverse effects on quality of life. ${ }^{1}$ Patients with low back pain (LBP) have been shown to have some limitations in spinal motion that compromises their function. Therefore, the ability to reliably measure and evaluate lumbar spine motion is essential in elucidating the pathophysiologies of various musculoskeletal disorders, such as LBP. ${ }^{17}$ The anatomy and function of the lumbar spine is complex and, therefore, requires a measurement technique that can record 3-dimensional (3-D) movements. Radiological imaging, including X-ray, fluoroscopy, and 3-D magnetic resonance imaging, are precise and accurate techniques that can evaluate intersegmental movement of spinal vertebrae. ${ }^{18,19}$ However, these invasive methods could be harmful to patients. ${ }^{18}$ Although electromagnetic tracking systems are a better alternative and would be a suitable technique for assessing functional activities (eg, gait, STS) in a clinical setting, the quantitative analysis of functional activities using optical motion analysis systems is well established, and has been used in clinical contexts for several decades in order to help diagnose, plan treatment, and assess treatment outcomes. ${ }^{18}$

Electromagnetic motion analysis and optical 3-D motion analysis systems are used for measuring range of motion (ROM) of multiple joints simultaneously. Both systems utilize markers for taking measurements, and it has been shown that they are highly accurate. ${ }^{20}$ The electromagnetic motion analysis system (eg, FasTrak) is a noninvasive electromagnetic measuring instrument that tracks the positions of sensors relative to a source in 3 dimensions. ${ }^{21}$ The optical 3-D motion analysis system (marker-based system) uses spherical retroreflective markers that can be identified by the 
cameras. The system outputs 3-D coordinates of detected markers usually at 100 to 120 frames per second. ${ }^{22}$ Although high accuracy and the ability of multiple simultaneous ROM measurements are the main advantages of optical 3-D motion analysis systems, some disadvantages should be considered. High cost and potential influence of soft tissue artifact are the main disadvantages of markerbased systems. ${ }^{23}$ The placement of markers on the skin overlying the spinous processes of the spinal column provides a noninvasive approach to measure dynamic movement of the spine during daily activities. It is also important to note that the difficulty in locating relevant anatomical landmarks to effectively define axial rotation in the transverse plane limits the analysis of lumbar spine kinematics to the frontal and sagittal planes using this approach for measuring functional tasks. ${ }^{18,24}$ Nevertheless, Shum et al $^{1}$ reported that the magnitude of movements out of the sagittal plane during STS, and its reverse, are very small and can be neglected.

Although there are several noninvasive approaches reported within the literature, and the review of all these technologies are beyond the scope of this article, motion analysis systems are generally accepted to be the "gold standard" for STS, gait, and movement analysis. ${ }^{18}$ Therefore, the purpose of this systematic review is to critically investigate published literature to assess the kinematics of the spine during STS task in patients with LBP and healthy young adult participants using motion analysis systems (electromagnetic and marker based). It is hoped that this systematic review will be helpful in further understanding the kinematics of the spine during STS.

\section{Methods}

\section{Scope and Boundaries}

This review intended to examine the methodological considerations for 2-dimensional and 3-D analysis of spinal movements using motion analysis systems. Areas for review included study and participant characteristics, motion analysis system, marker/ sensor design and placement, kinematic model description, data collection procedures, and outcome measures (ie, ROM, velocity, coordination, etc). This review did not critically analyze the mathematical procedures and algorithms used for maker detection.

\section{Search Strategy}

The methods adopted for this review were compliant with the recommended Preferred Reporting Items for Systematic Review and Meta-Analysis checklist guidelines for systematic reviews. Moreover, the Preferred Reporting Items for Systematic Review and Meta-Analysis flow diagram was used to describe the number of primary studies that were included and excluded in each stage of the selection process (Figure 1). A single reviewer (M.R.P.) searched in electronic databases: PubMed/MEDLINE (National Library of Medicine), Scopus, ScienceDirect, and Google Scholar were searched, corresponding to the period from January 2002 to February 2017 (15 y). This period of time was selected for searching because before this period, most of STS studies used other instruments rather than optoelectronic motion analysis systems or electromagnetic sensors, such as light-emitting diodes, ${ }^{25}$ simple video camera, ${ }^{26}$ electrogoniometers,${ }^{27}$ and so forth. Details of the PubMed database search syntax were as follows:

("sit-to-stand" OR "sit to stand" OR "chair* rise*" OR "chairrise" OR "chair* stand*" OR "stand* up") AND ("kinematic*" OR "biomechanic*") AND ("spin*" OR "trunk" OR “torso*” OR "back”) AND 2002/01/01:2017/02/31[dp].

The syntax of this review was a combination of medical subject headings terms and free text words. The Boolean operators AND and OR were used, alongside phrase searching. Wildcards

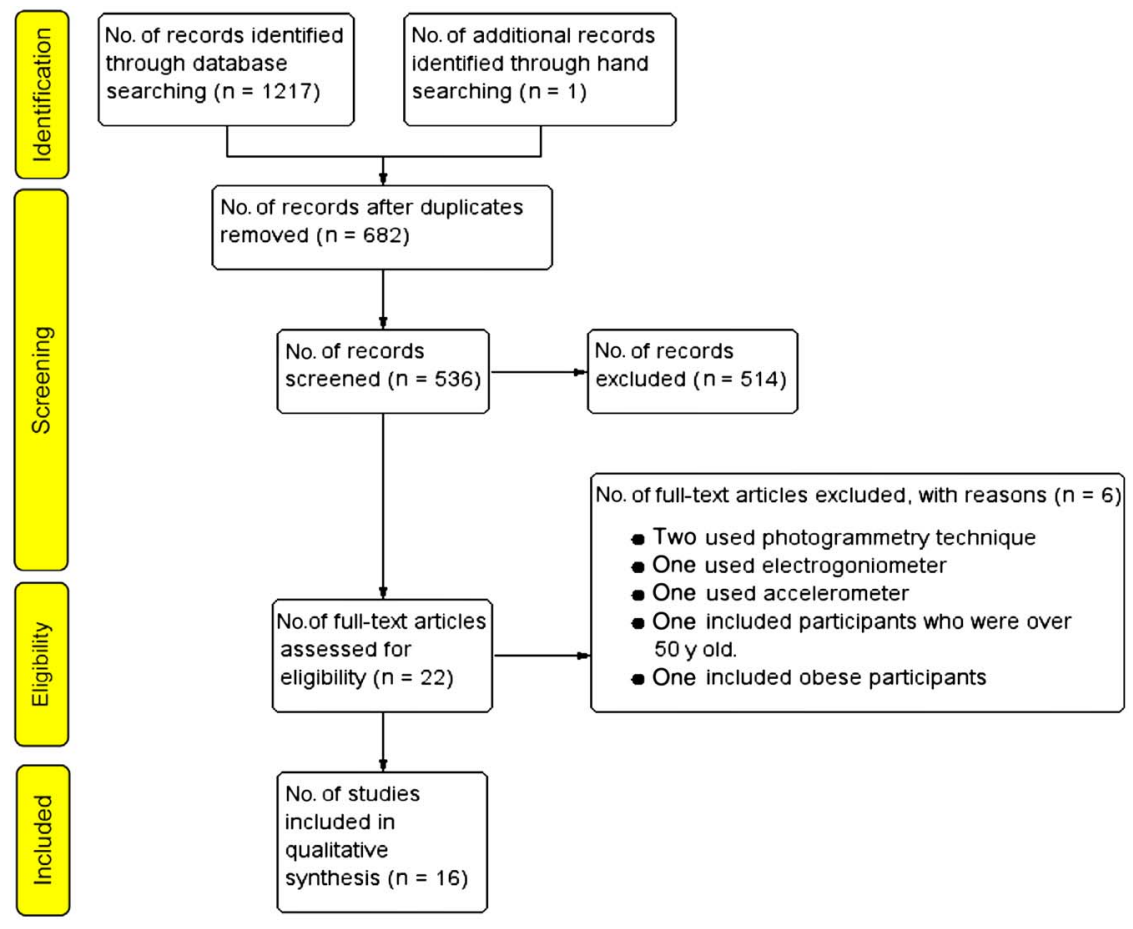

Figure 1 - Preferred Reporting Items for Systematic Review and Meta-Analysis flowchart of the study. 
and truncations were also used to enable the search to retrieve all possible variations of a specific root word. To optimize the strategy for each of the other databases, appropriate changes were made in the basic search strategy. Moreover, a hand search through a list of references of included studies was conducted to identify other eligible studies.

\section{Inclusion Criteria}

At the completion of the search, all references were transferred into EndNote, and duplicates were removed. Two reviewers (M.R.P. and R.B.) screened titles and abstracts of all primary articles that meet the search strategy in order to determine studies eligible for inclusion. If insufficient information was available in the title and abstract of an article, a full-text evaluation was undertaken. Then, the same 2 reviewers independently evaluated the full text of potentially relevant nonduplicated articles. Conflicts were resolved by discussion to reach consensus. In addition, it was planned that major discrepancies unable to be resolved by the reviewers would be taken to a third party (I.E.T.) for resolution. The following parameters were used to include the articles: Participants, Interventions/Diagnoses, Comparisons, and Outcomes criteria:

(1) Study design: Observational (case-control and crosssectional) studies published in peer-reviewed journals with full text available in English; results obtained from theses/ dissertations, conference proceedings, abstracts, and websites were excluded. In addition, studies were excluded if they investigated the effects of assisted devices or any other intervention.

(2) Participants and diagnoses: Studies in which participants were either healthy adults (mean age between 18 and $50 \mathrm{y}$ ) without functional limitations or patients with LBP. Other pathologies including spinal cord injury, Parkinson's disease, stroke survivors, multiple sclerosis, arthroplasty, amputation, and so forth, were excluded.

(3) Comparisons: Studies in which the kinematics of patients with LBP were compared with healthy control participants. Studies in which only healthy participants (without a control group) were recruited, were also included for this review.

(4) Outcomes: Studies in which one or more of the following outcomes were assessed: ROM in the cardinal planes, intersegmental motions, velocity, and spine coordination.

(5) Studies in which STS was assessed using a motion analysis system (electromagnetic or marker-based or inertial sensors) with no restrictions on methodology procedures (ie, rising speed, chair/stool height, sitting position).

\section{Methodological Quality Assessment}

The methodological quality of the included studies was assessed using a modified quality assessment tool developed around the major research aims. ${ }^{28,29}$ The quality assessment criteria included 13 appraisal questions and were specifically designed for assessing methodological procedures related to kinematic modeling and the reproducibility of a marker set configuration. ${ }^{28}$ Two items (10 and 15) were added to the original checklist, ${ }^{29}$ and 1 item (5) was modified. Item 5 was modified to "Is the spine (cervical and/or thoracic and/or lumbar) segment clearly stated?" Item 10 was added as "Were movement tasks clearly defined?" 29 In addition, item 15 was added as "Were conclusions drawn from the study clearly stated?" 29 (Table 1). Each item was scored as follows:

\section{Table 1 Assessment of Research Quality ${ }^{a}$}

1 Are the research objectives or aims clearly stated?

2 Is the study clearly described?

3 Are appropriate subject information and anthropometric details provided?

4 Are the marker/sensors locations accurately described?

5 Is the spine (cervical and/or thoracic and/or lumbar) segment clearly stated?

6 Is the reference position used to define anatomical frames reported?

7 Is the motion analysis equipment and set-up clearly described?

8 Are the segment coordinate systems clearly defined?

9 Are the model properties clearly defined for all joints (e.g. degrees of freedom)?

10 Were movement tasks clearly defined?

11 Are the methods used to describe the axes and order of rotations clearly described or referenced appropriately?

12 Are appropriate variability/reliability/repeatability procedures documented and reported?

13 Are the main outcomes of the study stated?

14 Are the limitations of the study clearly described?

15 Were conclusions drawn from the study clearly stated?

Note: Adapted from Bishop et al. ${ }^{28}$

${ }^{\mathrm{a}}$ Items were scored as follows: $2=$ yes; $1=$ limited detail; $0=$ no.

$2=$ yes, $1=$ limited detail, and $0=$ no. An article was deemed high quality if the total score was $\geq 24 / 30$ (cutoff point $=80 \%$ ). ${ }^{18,28,29}$

The 2 reviewers (M.R.P. and R.B.) independently assessed the quality of all included studies. The agreement between the 2 reviewers was calculated using Cohen's coefficient kappa (kappa: 0-.29= week agreement, .30-.59= moderate agreement, $.60-.89=$ good agreement, and $.90-1=$ optimal agreement). ${ }^{30}$ The results demonstrated that good agreement was present between the 2 reviewers (Cohen's kappa $\pm \mathrm{SE}$ was $.71 \pm .24$ ).

\section{Risk of Bias Assessment}

The risk of bias was analyzed for all individual studies using a checklist developed by the Grading of Recommendations Assessment, Development and Evaluation working group. ${ }^{31}$ The risk of bias was classified as "high" or "low" or "unclear" if there was an insufficient description in the original reports. ${ }^{32}$ One reviewer (M.R.P.) evaluated the risk of bias of each included study using the Grading of Recommendations Assessment, Development and Evaluation checklist for observational studies.

\section{Data Extraction and Analysis}

To carry out descriptive analyses, data were independently extracted by the 2 reviewers (M.R.P. and R.B.) from the identified studies. The extracted data included the description of study characteristics (first author's name, year of publication, country in which the study was performed, and size of the sample); the description of study participants (number, gender, mean age, body mass index, and status of health); the description and characteristics of the motion analysis system used, alongside markers/sensors, measurement frequency, test procedures, kinematic outcome measures; and the conclusion.

Meta-analysis was not performed because the included studies were heterogeneous and methodologically different (procedures 
and instrumentation). Therefore, this review focused only on description and qualitative synthesis of the identified studies.

\section{Results}

\section{Identification of Studies}

A total of 1218 studies were identified through the electronic database searches (Figure 1). After exclusion of duplicates and review of titles and abstracts, 22 studies were considered eligible for inclusion in our review. One study included participants who were more than 50 years old, ${ }^{33} 2$ studies used accelerometer or electrogoniometer, ${ }^{34,35}$ and 2 studies utilized photogrammetry techniques. ${ }^{36,37}$ Furthermore, 1 study recruited obese participants. $^{38}$ A hand search of references provided in the included studies identified 1 additional article. ${ }^{39}$ Thus, a total of 16 studies were included in this systematic review. ${ }^{1,24,39-52}$

\section{Quality Assessment}

A summary of the quality assessment of the reviewed articles is presented in Figure 2A. Using an approach proposed by Bishop et al, ${ }^{28}$ information required to sufficiently answer questions 6,8 , and 14 was not consistently provided in the articles included for review, and this was represented by a median score of $\leq 1$ (Figure 2B). From the 16 articles reviewed, 10 articles were deemed to be high quality (Figure 2A). ${ }^{1,24,39,40,43,46-50}$

\section{Risk of Bias in Included Studies}

Following the assessment of risk of bias, the results indicated that 4 studies failed to develop appropriate eligibility criteria. ${ }^{41,45,51,52}$ One study reported unclear information about the eligibility criteria as it only mentioned that healthy participants were included if they had no history of major musculoskeletal issues. ${ }^{43}$ All included studies that compared patients with LBP with healthy participants did not match known prognostic factors between patients with LBP and their controls. ${ }^{1,42,46-48,50,51}$ Incomplete or absent reporting of some outcomes was detected in 1 study. ${ }^{51}$ Shafizadeh $^{51}$ did not provide sufficient information about the differences of coordination values between patients with LBP and healthy participants during the second half of stand-to-sit movement. Finally, no study indicated participants' attrition (loss to follow-up). Figure 3 summarizes the risk of bias of the included studies.

\section{Overview of Participant Characteristics}

Table 2 provides a summary of the total number of participants recruited, along with their health status, gender, and age. The majority of studies included participants without a history of LBP. Both participants with and without LBP were included in 5 studies. ${ }^{1,46,48,50,51}$ In most of the included studies, LBP was defined as a pain on the lumbar region for a period of 0 to 12 months without sciatica and neurologic deficits. ${ }^{46,48,50}$ Moreover, Shum et $\mathrm{al}^{1}$ included subacute LBP ( $7 \mathrm{~d}$ to $12 \mathrm{wk}$ ) participants with and without a positive straight leg raising test. Four studies did not provide information on gender, ${ }^{1,24,42,46}$ whereas 3 studies examined only male participants ${ }^{41,49,52}$ and 1 study included only female participants. ${ }^{39}$ Parkinson et $\mathrm{al}^{47}$ assessed gender separately to show between-gender differences during STS task. The mean age of included studies population at baseline ranged from 20.1 to 46.2 years. None of the included studies clearly justified their sample size.

\section{Methodology Considerations and Outcome Measures}

The literature reports a wide range of models for spine segments. Two studies considered the whole-trunk kinematics during STS, ${ }^{41,48}$ whereas the others evaluated the different parts of the spine during STS and its reverse. In Johnson and Van Emmerik' ${ }^{45}$ study, no precise information has been available regarding the spinal modeling. Moreover, Slaboda et $\mathrm{al}^{42}$ and Kouta et $\mathrm{al}^{41}$ reported only head-arm-trunk and head segments kinematics in participants without LBP and did not provide further details about the kinematic model used for the assessment of STS. The majority of the included studies evaluated the kinematics of the spine in the sagittal plane, whereas some studies investigated the kinematics in the frontal and transverse planes. ${ }^{39,46,48}$ Christe et al $^{50}$ revealed that sagittal-plane angle of the lumbar and thoracic regions was
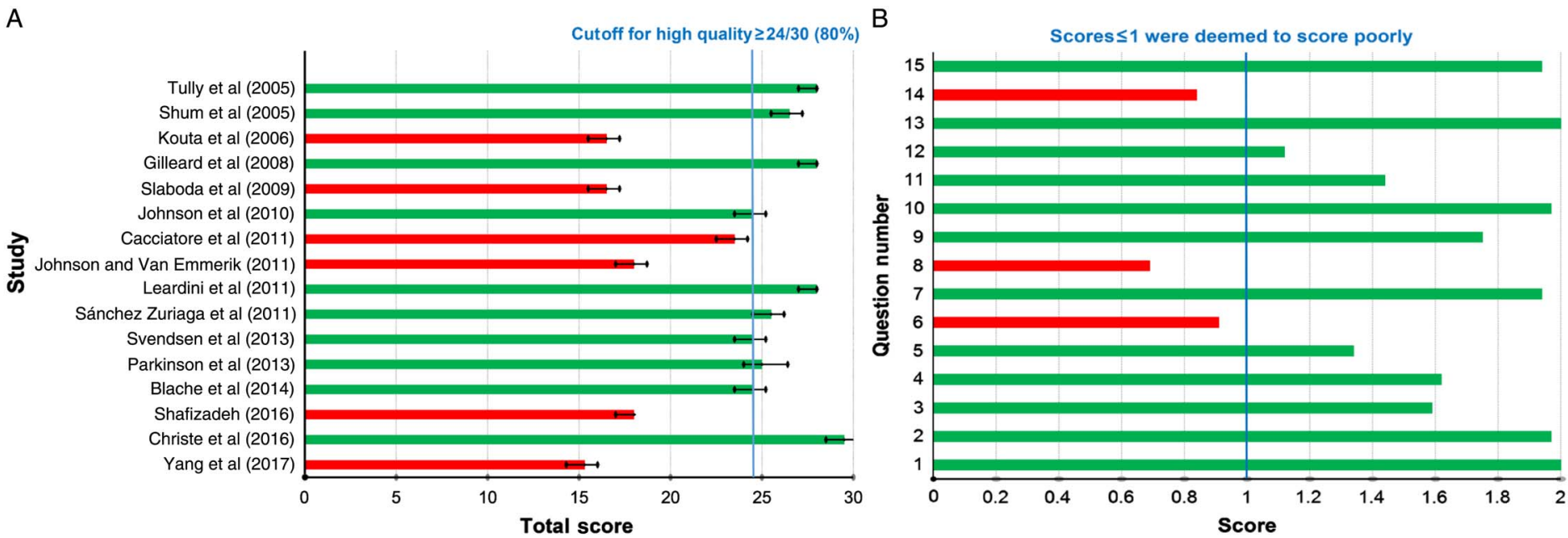

Figure 2 - Quality assessment results. (A) The median score for each article as judged by the 2 reviewers. The light gray (red in online) rows are indicative of those studies deemed not high quality (ie, a mean score $<24 / 30$ ). (B) The median score for each question in the appraisal tool. The light gray (red in online) rows are indicative of those questions that were poorly addressed (ie, a median score $\leq 1$ ). 


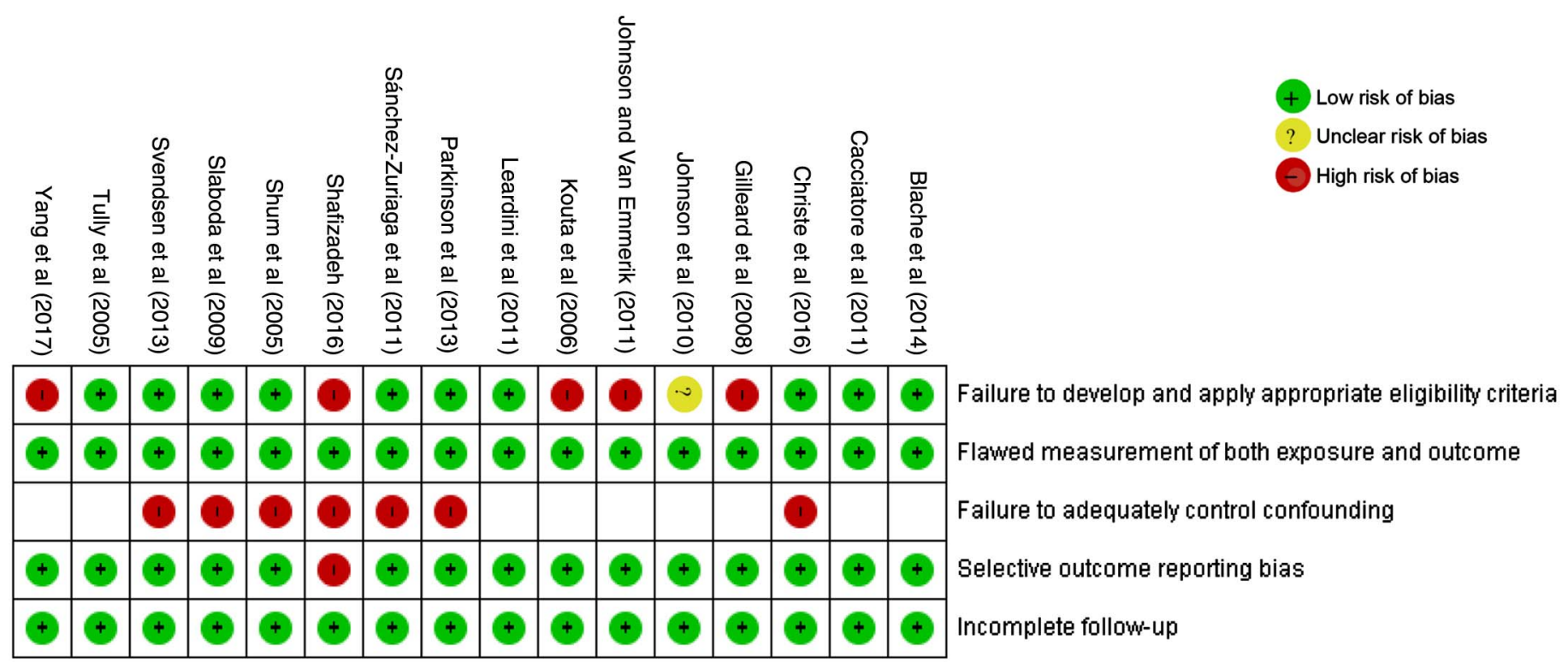

Figure 3 - Risk of bias summary for kinematic parameters. Because 9 studies $24,39-41,43-45,49,52$ included only 1 group of participants, therefore, it was not possible to assess the limitation of controlling confounding factors in these studies (empty boxes).

significantly limited in nonspecific CLBP participants compared with healthy adults. Likewise, Shum et $\mathrm{al}^{1}$ reported limited lumbar spine sagittal-plane ROM in subacute LBP patients with and without a positive straight leg raising. However, Svendsen et $\mathrm{al}^{48}$ found no significant differences in overall trunk angle between patients with LBP and healthy participants. Two studies mentioned that the trunk as a single segment could not adequately represent the spine kinematics. ${ }^{47,50}$ Parkinson et $\mathrm{al}^{47}$ reported that healthy males and females represented different lumbar spine sagittal-plane ROM during STS. Furthermore, Christe et al $^{50}$ indicated that the kinematics of the spine are different in the upper and lower parts of the lumbar and thoracic in participants with nonspecific CLBP and without LBP. They showed that patients with nonspecific CLBP performed STS with less spinal movement in the lumbar, but also in the thoracic regions. ${ }^{50}$ It has been demonstrated that the trunk segment displaces in the frontal and transverse planes during STS in patients with $\mathrm{LBP}^{48}$ and healthy participants. ${ }^{39,48}$ Large intersegmental motion over the 3 anatomical planes has been reported by Leardini et $\mathrm{al}^{24}$ The effects of seat height and 3 different foot positions on L5-S1 kinematics were investigated in Blache et $\mathrm{al}^{49}$ study. They reported that low-height seat and neutral foot position resulted in greater L5-S1 ROM. ${ }^{4}$ Shafizadeh $^{51}$ evaluated decomposition index values for the lumbar-hip joint pair as indicators of interjoint coordination. He concluded that patients with LBP had significantly higher decomposition indices relative to healthy participants during STS and stand-to-sit movements. ${ }^{51}$

Angular velocity of the spine was also assessed in 5 studies. ${ }^{1,41,42,45,50,51}$ Limited angular velocities in subacute LBP and patients with nonspecific CLBP have been reported by Shum et al ${ }^{1}$ and Christe et $\mathrm{al}^{50}$ studies, respectively. In addition, 1 study showed that head-on-trunk extension during STS decreased peak head velocity in the anteroposterior and vertical directions. ${ }^{45}$ Slaboda et $\mathrm{al}^{42}$ indicated that environment can alter the velocities of the trunk and head.

Accurate marker/sensor positions were provided in the majority of studies. ${ }^{1,24,39-41,43,44,46-48,50}$ Measurement frequencies used within the included studies ranged from 25 to $200 \mathrm{~Hz}$, and 4 studies used MATLAB software (The MathWorks Inc, Natick, MA) for data processing. ${ }^{24,43,45,50}$ The majority of the included studies described the procedure in detail.1,24,39-47,49-52 Stool's height, arm position, feet position, and rising speed were the most common items mentioned in the studies. Participants in all studies were asked to perform STS at a preferred speed. Number of STS trials ranged between 3 and 15 trials. However, 4 studies reported no information on the number of trials. ${ }^{24,40,41,47}$ Finally, reliability analyses were appropriately documented and reported in 9 studies. $^{1,24,39,40,46-50}$

\section{Discussion}

Assessment of spinal mobility is critical for estimating disability, evaluating outcomes, and guiding nonsurgical treatment approaches. ${ }^{53,54}$ Motion analysis systems can provide high accurate information of the spine kinematics during various tasks, and it has been reported that optical motion analysis systems are the golden standard in motion capture and analysis. ${ }^{55}$ In addition, motion analysis systems are noninvasive, allow for the repetition of the examination more times within a short period of time, and provide quantitative and 3-D data. The results of this review indicated that patients with LBP have limited sagittal-plane angle and smaller angular velocity compared with healthy participants. Decreased ROM may be due to pain, muscle spasm, muscles coactivation, or stiffness. Sung ${ }^{56}$ mentioned that coactivation of the paraspinal muscles is used to immobilize the lumbar spine as a protective strategy to avoid provocation of pain. In addition, increased spinal stiffness could alter movement patterns of patients with LBP in a harmful way and possibly increase sensitization of spinal and peripheral structures, which could adversely contribute to the chronicity of pain. ${ }^{50,57,58}$ In Shum et al's ${ }^{1}$ study, it was found that patients with subacute LBP had a significant reduction in velocity in both the lumbar spine and hip joints, and took a longer time to complete STS and its reverse movements. They mentioned that patients with LBP probably decrease trunk velocities and 


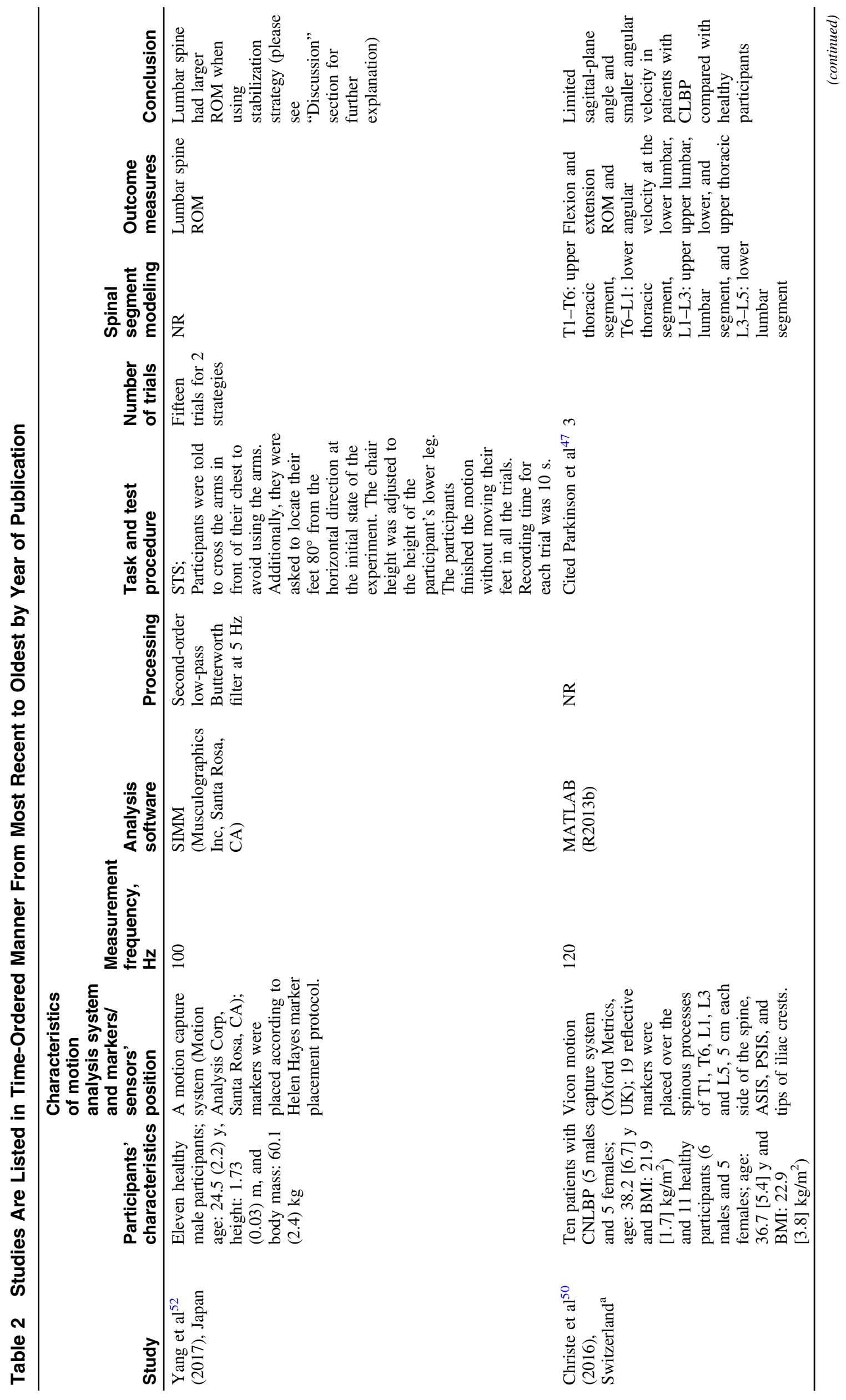




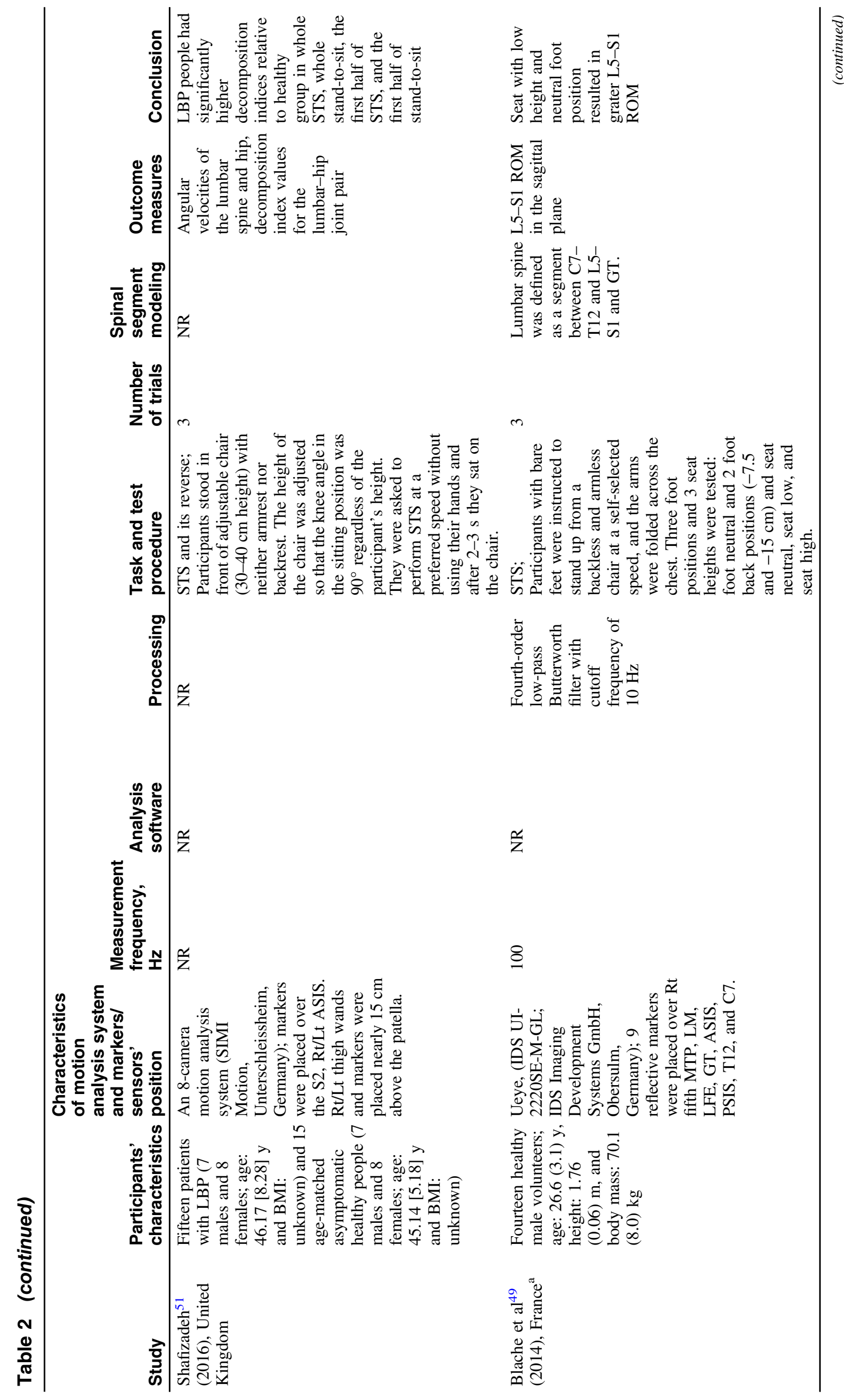




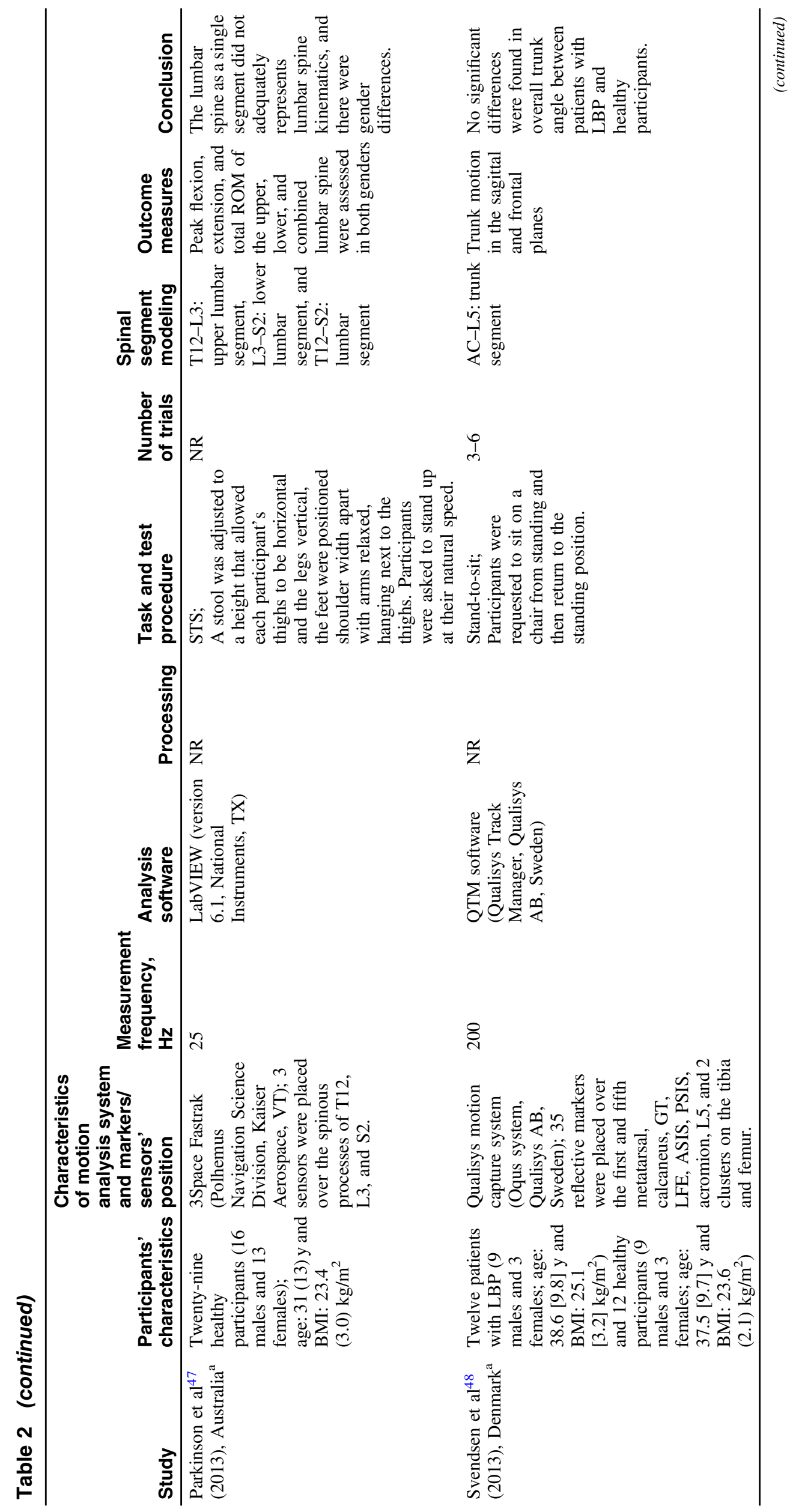




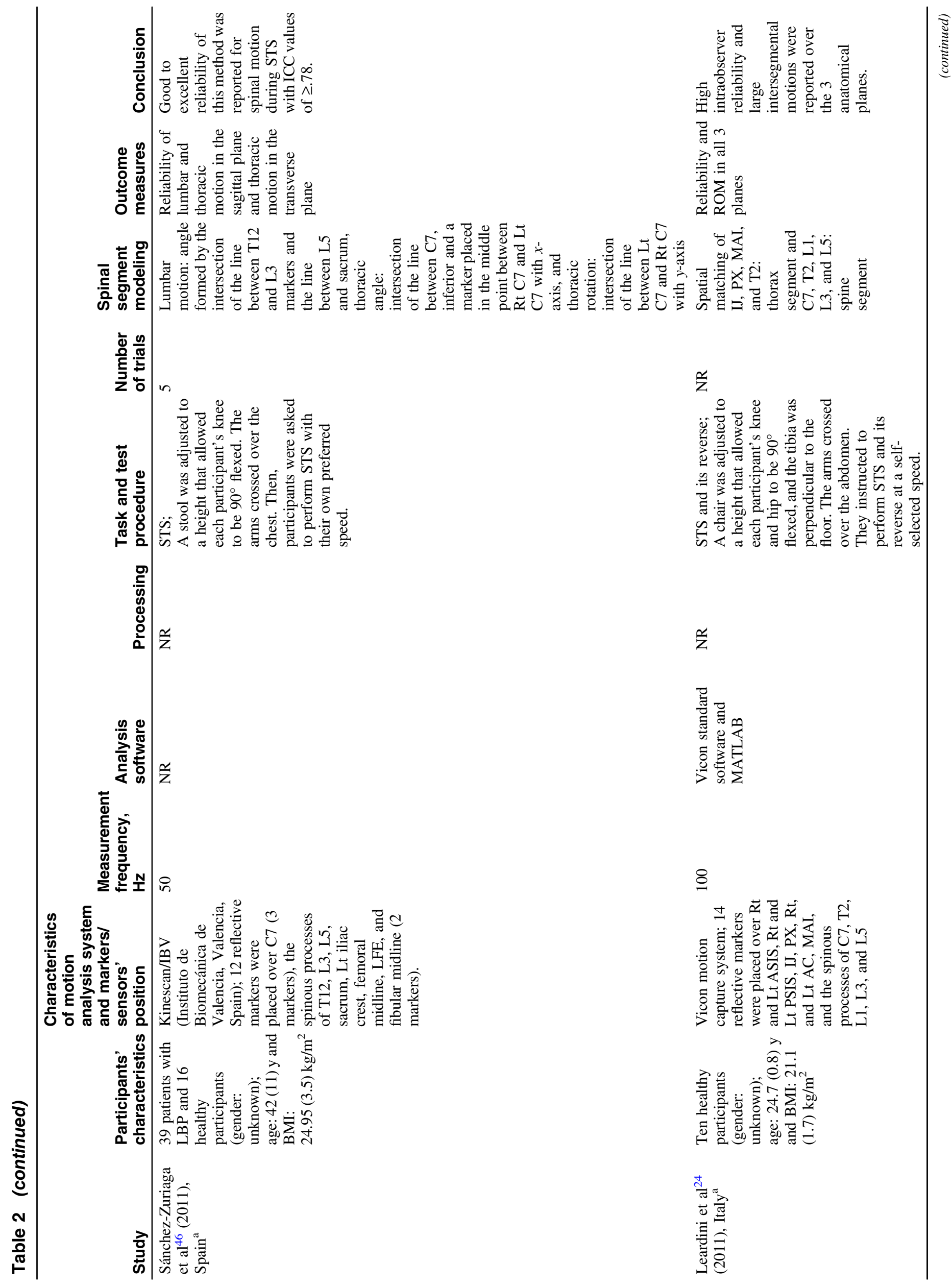




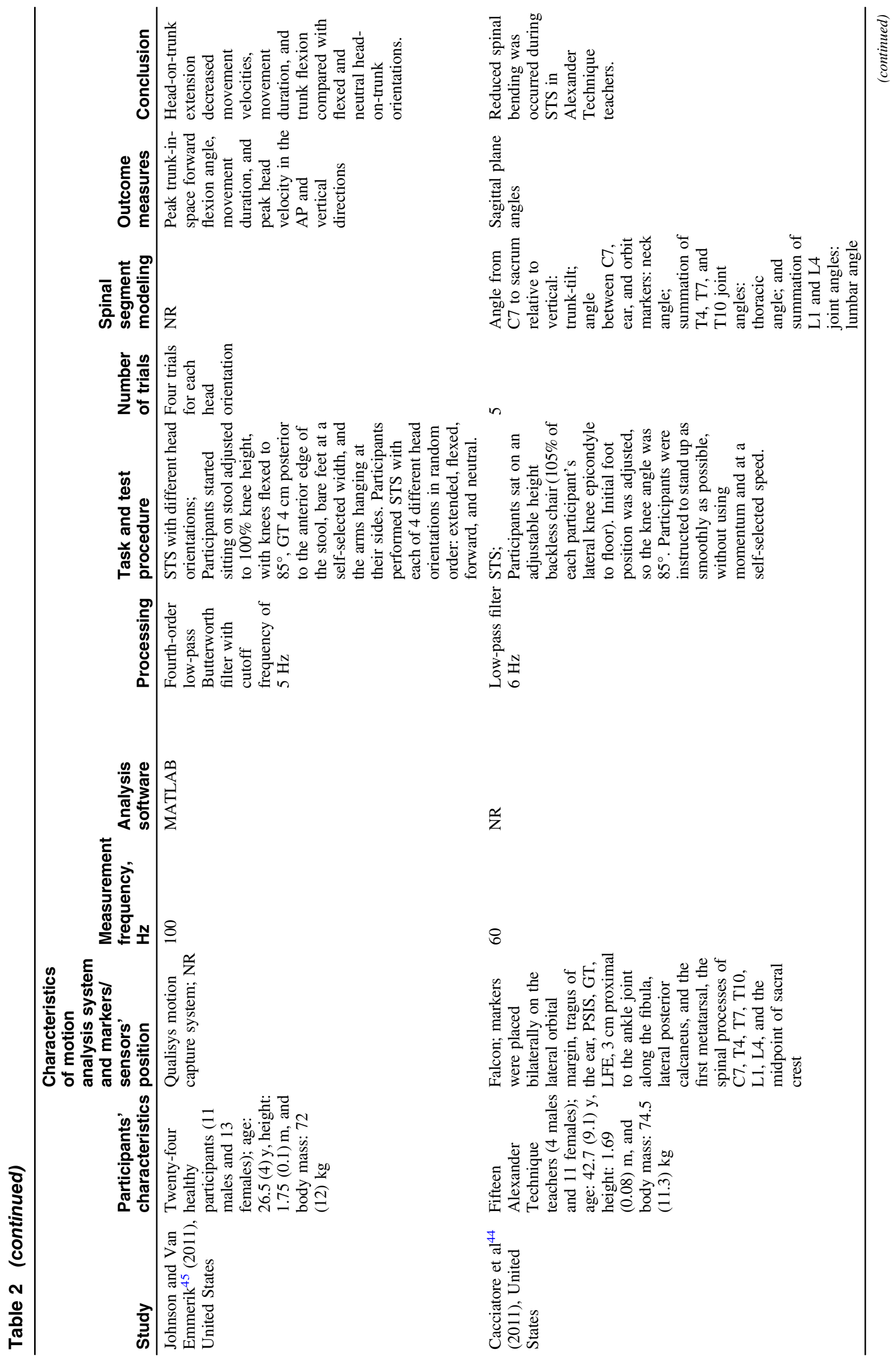




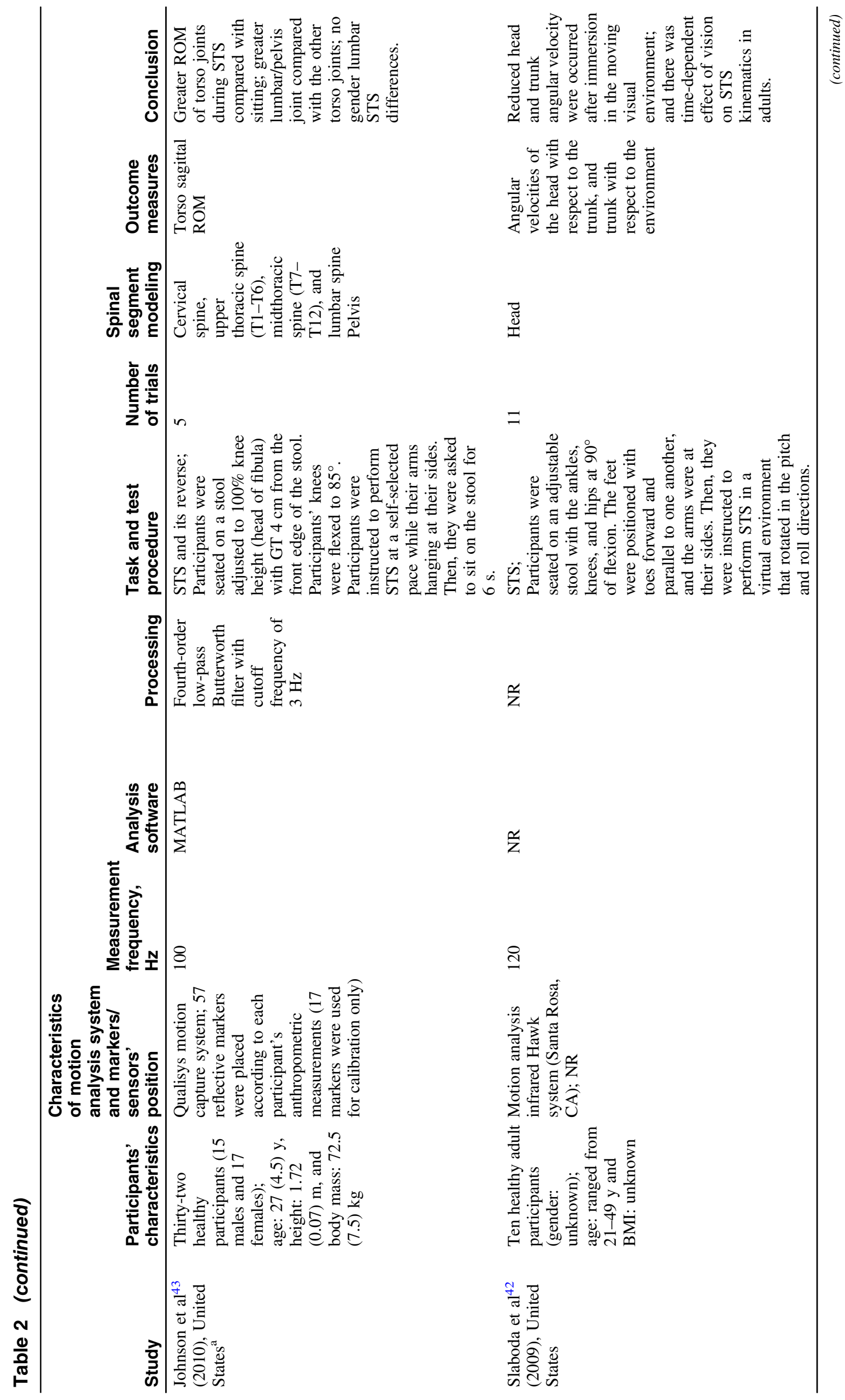




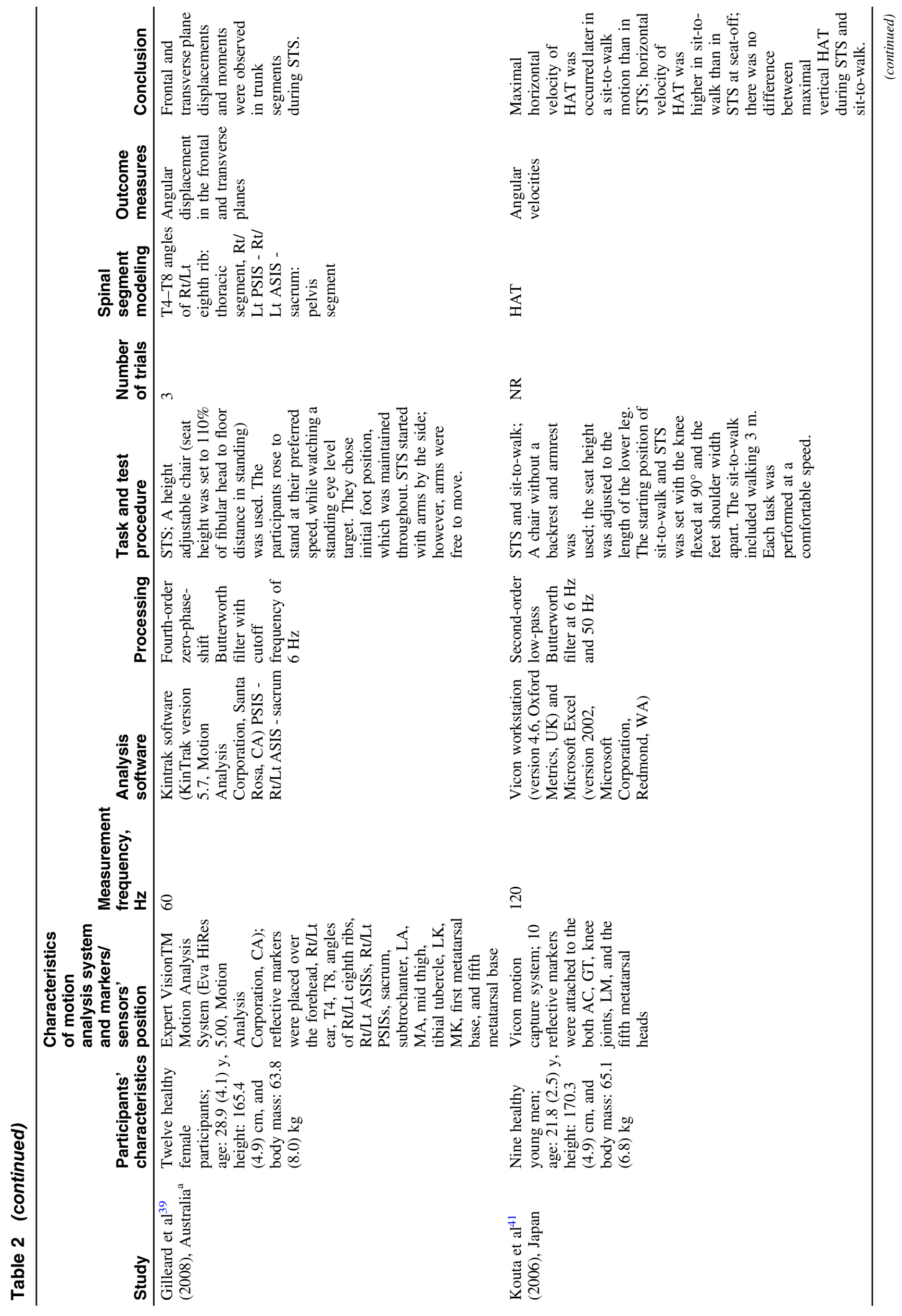




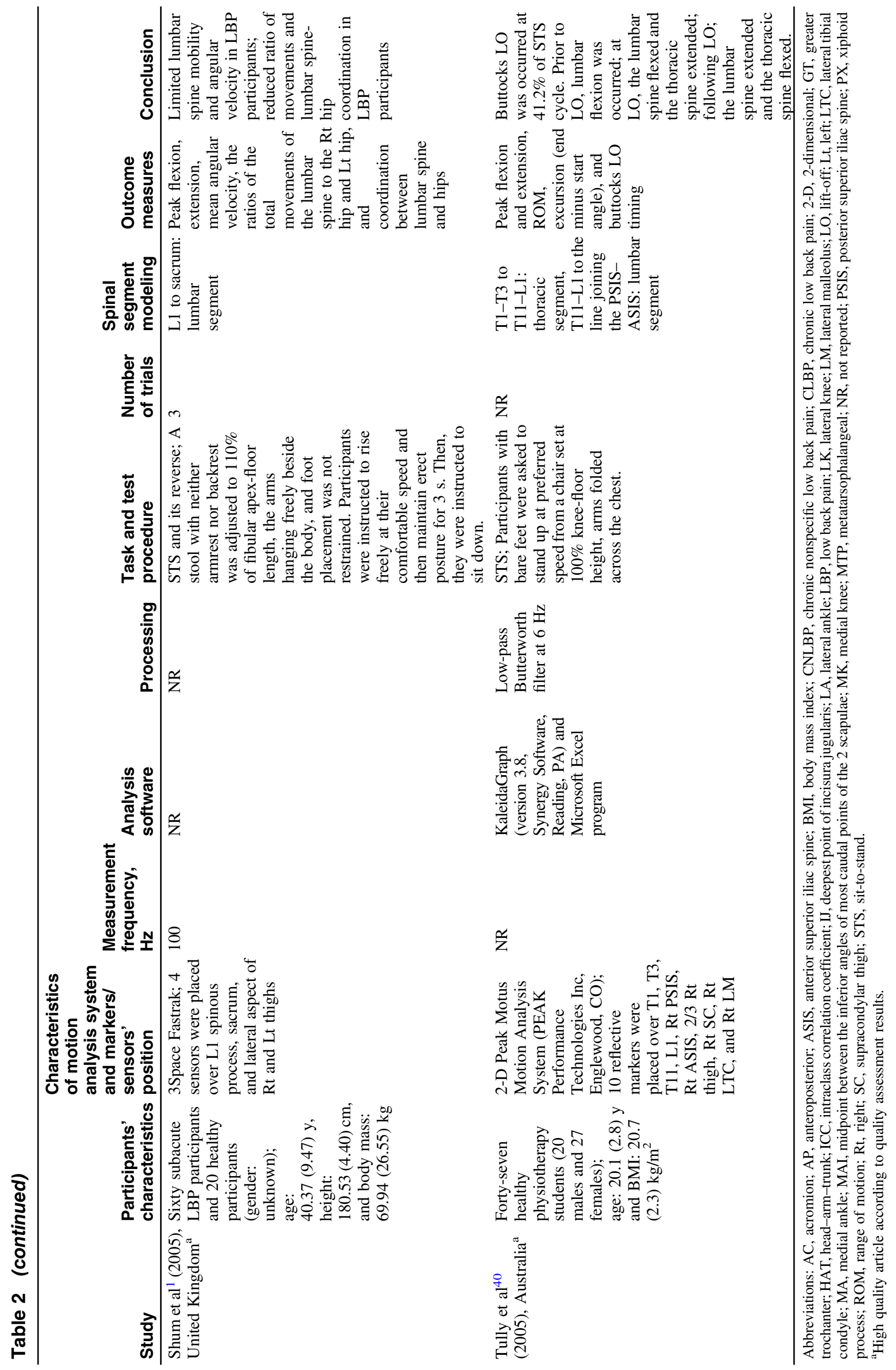


acceleration to avoid exacerbation of pain caused by muscle contraction and high levels of acceleration. ${ }^{1}$ Shum et al findings were consistent with the result of a previous study, in which LBP had more significant effects on trunk velocities than trunk mobility. ${ }^{59}$ However, Crosbie et $\mathrm{al}^{60}$ reported altered kinematics in recurrent patients with LBP who were in a pain-free period at the time of experiment. The main reason could be related to changes of spinal motor behavior due to reorganization of motor task planning. ${ }^{61-63}$ Previous studies have recommended differentiating the lower and upper lumbar spine regions and evaluating thoracic kinematics for the assessment of STS. ${ }^{24,47,60}$ They believe that analyzing STS using a multisegment lumbar model assists in achieving a better understanding of spine kinematics compared with a single-segment lumbar model. Christe et $\mathrm{al}^{50}$ indicated that, in patients with nonspecific CLBP, flexion ROM reduction occurred principally at the upper lumbar segment. As STS movement is often described as a painful maneuver by patients with LBP, ${ }^{1,16,64}$ Christe et al ${ }^{50}$ suggested that pain is related to the rapid changes from a flexion to an extension posture required by this task. Moreover, interjoint discoordination is present in patients with LBP compared with asymptomatic individuals. ${ }^{1,51}$ Shum et al ${ }^{1}$ used relative phase angle, and Shafizadeh ${ }^{51}$ used decomposition index to assess lumbar-hip coordination. Phase angle is defined as the inverse tangent of angular velocity/angular displacement. ${ }^{1,65}$ The relative phase angle between 2 joints is quantified by subtracting the phase angle of one from the other.,65 In addition, decomposition index for the lumbar-hip joint pair is defined when one joint is moving while the other joint is paused. ${ }^{51}$ The results of 2 studies showed that lumbar-hip coordination is more separated in time and more variable in LBP people. ${ }^{1,51}$ Reduction in the angular velocity of both lumbar and hip joints during STS and its reverse, ${ }^{1,59}$ and difficulty in transferring the muscle force from the pelvis to the lower limbs, ${ }^{51,66}$ can cause lumbar-hip discoordination. A point worth mentioning here is that the reliability of marker/sensor placement techniques for evaluating lumbar-hip coordination using these methods has not been well established in the literature, and more studies are warranted to investigate the reliability of various marker/sensor placement techniques for assessing lumbar-hip coordination using the abovementioned methods.

In studying STS, it is essential to know that Parkinson et $\mathrm{al}^{47}$ demonstrated that there were significant between-gender differences when the lumbar spine was modeled as 2 segments: the lower lumbar and upper lumbar regions. In the present study, healthy female participants showed greater peak flexion ROM in the upper lumbar segment, whereas peak flexion ROM in healthy male participants was greater in the lower segment. ${ }^{47}$ Furthermore, no between-gender differences were found when the lumbar spine was modeled as a single region. ${ }^{47}$

Hughes et $\mathrm{al}^{3}$ described 3 strategies (momentum transfer, stabilization, and hybrid) used in humans STS activity that generate different movements. They reported that younger individuals tend to use the momentum transfer strategy that utilizes the momentum to lift up their body. ${ }^{52}$ However, the elderly persons tend to use the stabilization strategy, in which they carry their center of mass first on their feet and then they move upward. ${ }^{52}$ The hybrid strategy is in the middle between momentum transfer and stabilization strategies. ${ }^{52}$ Yang et $\mathrm{al}^{52}$ found that the lumbar spine had larger ROM when using a stabilization strategy. They also showed that the subjects need to flex their body more to move their center of mass forward when using a stabilization strategy. ${ }^{52}$ The present study showed that 4 muscle synergies could generate STS motion, and the study subjects adaptively changed the start time of a certain synergy to achieve different strategies. ${ }^{52}$

One study suggested that low seat height can increase L5-S1 joint ROM during STS. ${ }^{49}$ Besides, it has been reported that low seat height can result in an increase in the amount of paraspinal muscles activation and in the L5-S1 peak net joint torque compared with high and neutral seat heights. ${ }^{49}$ Hence, seat height modification in patients with LBP may greatly improve their quality of daily activities. Kuo et $\mathrm{al}^{67}$ mentioned that a high seat position is less demanding for the lumbar spine.

Slaboda et $\mathrm{al}^{42}$ showed that adult subjects, when seated in a moving visual environment, compensated for the constantly changing environment by decreasing the head and trunk angular velocities, as well as head and trunk center of mass velocities, from the initiation of STS motion to the standing position. During STS, the center of mass is displaced from a seated position to a standing position through the coordinated motion of the upper and lower body segments. ${ }^{68}$ If adults altered the displacement in response to the visual field motion, STS kinematics would be changed, which could potentially cause instability if the center of mass moved away from the lower limbs. In decreasing velocity, adults moved their bodies the same distance in the sagittal plane, but at a slower speed. This strategy may be used by adults to control the location of the center of mass when they realize that the visual environment is moving separately from their own movement. ${ }^{42}$ In another study, Kouta et $\mathrm{al}^{41}$ compared 2 common activities (STS vs sit-to-walk) among healthy young adults. The results of their study showed that the center of gravity moved higher in STS than sit to walk and moved further forward in sit-to-walk task. ${ }^{41}$ Finally, Kouta et $\mathrm{al}^{41}$ concluded that the forward translation of the center of gravity during sit-to-walk activity requires more advanced motor control ability.

Intravariability and intervariability is inherent in all biological systems ${ }^{69}$ and is, therefore, an important parameter to measure. ${ }^{18}$ The majority of the included studies recorded between 3 and 15 trials, and linear statistical methods such as SD, intraclass correlations, and coefficients of variation were utilized to analyze the variance of ROM values between trials and individuals. Several issues can affect reliability, including accurate identification of anatomical landmarks, familiarity of subjects with testing procedures, firm placement of markers or sensors over the skin, number of trial repetitions, sample size, experience of examiner(s), calibration of measurement system, and the region of the spine and type of ROM being examined..$^{70}$ All these parameters should be considered during the assessment of STS using a motion analysis system.

\section{Study Limitations and Future Research Recommendation}

The present study can be criticized by some limitations. First, only the studies published in peer-review journals were reviewed in this study, and as in other reviews, a publication bias may have occurred. Second, a language bias is possible as only those studies that were available as full text in English were included. Third, only the kinematics of healthy adults and patients with LBP were assessed in this review. Further reviews can evaluate the kinematics of elderly people or patients with a specific pathology (such as stroke, Parkinson's disease, etc) during STS. Moreover, future original researches can investigate the effects of physiotherapy treatment programs on the kinematics of the spine in patients with LBP. ${ }^{1,65}$ 


\section{Conclusion}

From the 16 studies included in this review, 10 studies were deemed high quality. Six studies had lesser quality based on the quality assessment results; therefore, a definitive conclusion cannot be drawn from the results of those studies. However, the results of this review demonstrated that there are differences in the kinematics of the spine between healthy people and patients with LBP. Several studies recommended that in order to develop a better understanding of spine kinematics, the trunk should be analyzed as a multisegment. One study revealed that high seat height reduces loads on the lumbar spine and, therefore, improves daily activities performance of patients with LBP. Further studies in healthy and subclinical populations with LBP are necessary to develop a better understanding of spine kinematics during STS.

\section{Acknowledgments}

The authors received no funding in support of this review and declared that there are no competing interests regarding the publication of this review.

\section{References}

1. Shum GL, Crosbie J, Lee RY. Effect of low back pain on the kinematics and joint coordination of the lumbar spine and hip during sit-to-stand and stand-to-sit. Spine. 2005;30(17):1998-2004. PubMed ID: 16135992 doi:10.1097/01.brs.0000176195.16128.27

2. Fotoohabadi MR, Tully EA, Galea MP. Kinematics of rising from a chair: image-based analysis of the sagittal hip-spine movement pattern in elderly people who are healthy. Phys Ther. 2010;90(4): 561-571. PubMed ID: 20167645 doi:10.2522/ptj.20090093

3. Hughes M, Weiner D, Schenkman M, Long R, Studenski S. Chair rise strategies in the elderly. Clin Biomech. 1994;9(3):187-192. doi:10. 1016/0268-0033(94)90020-5

4. Hortobagyi T, Mizelle C, Beam S, DeVita P. Old adults perform activities of daily living near their maximal capabilities. J Gerontol A Biol Sci Med Sci. 2003;58(5):M453-M460. PubMed ID: 12730256 doi:10.1093/erona/58.5.M453

5. Weiner DK, Long R, Hughes MA, Chandler J, Studenski S. When older adults face the chair-rise challenge. A study of chair height availability and height-modified chair-rise performance in the elderly. $J$ Am Geriatr Soc. 1993;41(1):6-10. PubMed ID: 8418126 doi:10. 1111/j.1532-5415.1993.tb05939.x

6. Bohannon RW, Shove ME, Barreca SR, Masters LM, Sigouin CS. Five-repetition sit-to-stand test performance by community-dwelling adults: a preliminary investigation of times, determinants, and relationship with self-reported physical performance. Isokinet Exerc Sci. 2007;15(2):77-81.

7. Brodin E, Ljungman S, Sunnerhagen KS. Rising from a chair: a simple screening test for physical function in predialysis patients. Scand J Urol Nephrol. 2008;42(3):293-300. doi:10.1080/00365 590701797556

8. Janssen W, Bussmann J, Selles R, Koudstaal P, Ribbers G, Stam H. Recovery of the sit-to-stand movement after stroke: a longitudinal cohort study. Neurorehabil Neural Repair. 2010;24(8):763-769. PubMed ID: 20702392 doi:10.1177/1545968310363584

9. Tung FL, Yang YR, Lee CC, Wang RY. Balance outcomes after additional sit-to-stand training in subjects with stroke: a randomized controlled trial. Clin Rehabil. 2010;24(6):533-542. PubMed ID: 20410150 doi:10.1177/0269215509360751
10. Dall PM, Kerr A. Frequency of the sit to stand task: an observational study of free-living adults. Appl Ergon. 2010;41(1):58-61. PubMed ID: 19450792 doi:10.1016/j.apergo.2009.04.005

11. Hsieh CY, Pringle RK. Range of motion of the lumbar spine required for four activities of daily living. J Manipulative Physiol Ther. 1994;17(6):353-358.

12. Tiedemann A, Shimada H, Sherrington C, Murray S, Lord S. The comparative ability of eight functional mobility tests for predicting falls in community-dwelling older people. Age Ageing. 2008;37(4):430-435. PubMed ID: 18487264 doi:10.1093/ageing/ afn 100

13. Puthoff ML, Nielsen DH. Relationships among impairments in lowerextremity strength and power, functional limitations, and disability in older adults. Phys Ther. 2007;87(10):1334-1347. PubMed ID: 17684086 doi: $10.2522 /$ ptj. 20060176

14. Shepherd R, Carr J. Reflections on physiotherapy and the emerging science of movement rehabilitation. Aust J Physiother. 1994;40S: 39-47. doi:10.1016/S0004-9514(14)60622-X

15. Janssen WG, Bussmann HB, Stam HJ. Determinants of the sit-tostand movement: a review. Phys Ther. 2002;82(9):866-879. PubMed ID: 12201801

16. Andersson EI, Lin CC, Smeets RJ. Performance tests in people with chronic low back pain: responsiveness and minimal clinically important change. Spine. 2010;35(26):E1559-E1563. PubMed ID: 20634779 doi:10.1097/BRS.0b013e3181cea12e

17. Cox ME, Asselin S, Gracovetsky SA, et al. Relationship between functional evaluation measures and self-assessment in nonacute low back pain. Spine. 2000;25(14):1817-1826. PubMed ID: 10888951 doi:10.1097/00007632-200007150-00013

18. Needham R, Stebbins J, Chockalingam N. Three-dimensional kinematics of the lumbar spine during gait using marker-based systems: a systematic review. J Med Eng Technol. 2016;40(4):172-185. PubMed ID: 27011295 doi:10.3109/03091902.2016.1154616

19. Fujii R, Sakaura H, Mukai Y, et al. Kinematics of the lumbar spine in trunk rotation: in vivo three-dimensional analysis using magnetic resonance imaging. Eur Spine J. 2007;16(11):1867-1874. PubMed ID: 17549527 doi:10.1007/s00586-007-0373-3

20. Ehara Y, Fujimoto H, Miyazaki S, Tanaka S, Yamamoto S. Comparison of the performance of 3D camera systems. Gait Posture. 1995;3(3):166-169. doi:10.1016/0966-6362(95)99067-U

21. Kristjansson E, Dall'Alba P, Jull G. A study of five cervicocephalic relocation tests in three different subject groups. Clin Rehabil. 2003; 17(7):768-774. PubMed ID: 14606744 doi:10.1191/0269215503 cr676oa

22. Stone E, Skubic M. Evaluation of an inexpensive depth camera for in-home gait assessment. J Ambient Intell Smart Environ. 2011;3(4): 349-361.

23. Leardini A, Chiari L, Della Croce U, Cappozzo A. Human movement analysis using stereophotogrammetry. Part 3. Soft tissue artifact assessment and compensation. Gait Posture. 2005;21(2):212-225. PubMed ID: 15639400 doi:10.1016/j.gaitpost.2004.05.002

24. Leardini A, Biagi F, Merlo A, Belvedere C, Benedetti MG. Multisegment trunk kinematics during locomotion and elementary exercises. Clin Biomech. 2011;26(6):562-571. doi:10.1016/j.clinbiomech. 2011.01.015

25. Schenkman M, Berger RA, Riley PO, Mann RW, Hodge WA. Whole-body movements during rising to standing from sitting. Phys Ther. 1990;70(10):638-648. PubMed ID: 2217543 doi:10. $1093 / \mathrm{ptj} / 70.10 .638$

26. Jeng S-F, Schenkman M, Riley PO, Lin S-J. Reliability of a clinical kinematic assessment of the sit-to-stand movement. Phys Ther. 1990; 70(8):511-520. PubMed ID: 2374780 doi:10.1093/ptj/70.8.511 
27. Kerr KM, White JA, Barr DA, Mollan RAB. Analysis of the sit-standsit movement cycle in normal subjects. Clin Biomech. 1997;12(4): 236-245. doi:10.1016/S0268-0033(96)00077-0

28. Bishop C, Paul G, Thewlis D. Recommendations for the reporting of foot and ankle models. J Biomech. 2012;45(13):2185-2194. PubMed ID: 22832021 doi:10.1016/j.jbiomech.2012.06.019

29. Peters A, Galna B, Sangeux M, Morris M, Baker R. Quantification of soft tissue artifact in lower limb human motion analysis: a systematic review. Gait Posture. 2010;31(1):1-8. PubMed ID: 19853455 doi: 10.1016/j.gaitpost.2009.09.004

30. Cohen J. A coefficient of agreement for nominal scales. Educ Psychol Meas. 1960;20(1):37-46. doi:10.1177/001316446002000104

31. Guyatt GH, Oxman AD, Vist G, et al. GRADE guidelines: 4. Rating the quality of evidence-study limitations (risk of bias). J Clin Epidemiol. 2011;64(4):407-415. PubMed ID: 21247734 doi:10. 1016/j.jclinepi.2010.07.017

32. Higgins JP, Green S. Cochrane Handbook for Systematic Reviews of Interventions. Vol. 4. Hoboken, NJ: John Wiley \& Sons; 2011.

33. Duclos C, Nadeau S, Lecours J. Lateral trunk displacement and stability during sit-to-stand transfer in relation to foot placement in patients with hemiparesis. Neurorehabil Neural Repair. 2008; 22(6):715-722. PubMed ID: 18812434 doi:10.1177/1545968308 316000

34. Kim MH, Yi CH, Yoo WG, Choi BR. EMG and kinematics analysis of the trunk and lower extremity during the sit-to-stand task while wearing shoes with different heel heights in healthy young women. Hum Mov Sci. 2011;30(3):596-605. doi:10.1016/j.humov.2010. 09.003

35. Alqhtani RS, Jones MD, Theobald PS, Williams JM. Correlation of lumbar-hip kinematics between trunk flexion and other functional tasks. J Manipulative Physiol Therap. 2015;38(6):442-447. doi:10. 1016/j.jmpt.2015.05.001

36. Dreischarf M, Bergmann G, Wilke HJ, Rohlmann A. Different arm positions and the shape of the thoracic spine can explain contradictory results in the literature about spinal loads for sitting and standing. Spine. 2010;35(22):2015-2021. PubMed ID: 20959780 doi:10.1097/ BRS.0b013e3181d55d52

37. Peydro MF, López J, Cortés A, Vivas MJ, Garrido JD, Tortosa L. Kinetic and kinematic analysis of the "getting up from a chair" movement in patients with low back pain. Rehabilitacion. 2011; 45(2):99-105. doi:10.1016/j.rh.2011.01.001

38. Sibella F, Galli M, Romei M, Montesano A, Crivellini M. Biomechanical analysis of sit-to-stand movement in normal and obese subjects. Clin Biomech. 2003;18(8):745-750. doi:10.1016/S02680033(03)00144-X

39. Gilleard W, Crosbie J, Smith R. Rising to stand from a chair: symmetry, and frontal and transverse plane kinematics and kinetics. Gait Posture. 2008;27(1):8-15. PubMed ID: 17166719 doi:10.1016/ j.gaitpost.2006.11.002

40. Tully EA, Fotoohabadi MR, Galea MP. Sagittal spine and lower limb movement during sit-to-stand in healthy young subjects. Gait Posture. 2005;22(4):338-345. PubMed ID: 16274916 doi:10.1016/ j.gaitpost.2004.11.007

41. Kouta M, Shinkoda K, Kanemura N. Sit-to-walk versus sit-to-stand or gait initiation: biomechanical analysis of young men. $J$ Phys Ther Sci. 2006;18(2):201-206. doi:10.1589/jpts.18.201

42. Slaboda JC, Barton JE, Keshner EA. Influence of moving visual environment on sit-to-stand kinematics in children and adults. Percept Mot Skills. 2009;109(1):121-132. PubMed ID: 19831093 doi: 10.2466/pms.109.1.121-132

43. Johnson MB, Cacciatore TW, Hamill J, Van Emmerik RE. Multisegmental torso coordination during the transition from sitting to standing. Clini Biomech. 2010;25(3):199-205. doi:10.1016/ j.clinbiomech.2009.11.009

44. Cacciatore TW, Gurfinkel VS, Horak FB, Day BL. Prolonged weightshift and altered spinal coordination during sit-to-stand in practitioners of the Alexander Technique. Gait Posture. 2011;34(4): 496-501. PubMed ID: 21782443 doi:10.1016/j.gaitpost.2011.06.026

45. Johnson MB, Van Emmerik REA. Is head-on-trunk extension a proprioceptive mediator of postural control and sit-to-stand movement characteristics? J Mot Behav. 2011;43(6):491-498. PubMed ID: 22122272 doi:10.1080/00222895.2011.631954

46. Sánchez-Zuriaga D, López-Pascual J, Garrido-Jaén D, De Moya MFP, Prat-Pastor J. Reliability and validity of a new objective tool for low back pain functional assessment. Spine. 2011;36(16):12791288. PubMed ID: 21240051 doi:10.1097/BRS.0b013e3181f471d8

47. Parkinson S, Campbell A, Dankaerts W, Burnett A, O'sullivan P. Upper and lower lumbar segments move differently during sit-tostand. Man Ther. 2013;18(5):390-394. PubMed ID: 23473753 doi: 10.1016/j.math.2013.02.001

48. Svendsen JH, Svarrer H, Laessoe U, Vollenbroek-Hutten M, Madeleine P. Standardized activities of daily living in presence of sub-acute low-back pain: a pilot study. J Electromyogr Kinesiol. 2013;23(1): 159-165. PubMed ID: 22995335 doi:10.1016/j.jelekin.2012. 08.006

49. Blache Y, Pairot de Fontenay B, Monteil K. The effects of seat height and foot placement on lumbar spine load during sit-to-stand tasks. Ergonomics. 2014;57(11):1687-1695. PubMed ID: 25174255 doi: 10.1080/00140139.2014.948495

50. Christe G, Redhead L, Legrand T, Jolles BM, Favre J. Multi-segment analysis of spinal kinematics during sit-to-stand in patients with chronic low back pain. J Biomech. 2016;49(10):2060-2067. PubMed ID: 27262182 doi:10.1016/j.jbiomech.2016.05.015

51. Shafizadeh M. Movement coordination during sit-to-stand in low back pain people. Human Mov. 2016;17(2):107-111.

52. Yang N, An Q, Yamakawa H, Tamura Y, Yamashita A, Asama H. Muscle synergy structure using different strategies in human standing-up motion. Adv Robot. 2017;31(1-2):40-54. doi:10.1080/ 01691864.2016.1238781

53. Kulig K, Powers CM, Landel RF, et al. Segmental lumbar mobility in individuals with low back pain: in vivo assessment during manual and self-imposed motion using dynamic MRI. BMC Musculoskelet Disord. 2007;8(1):8. doi:10.1186/1471-2474-8-8

54. Rainville J, Sobel JB, Hartigan C. Comparison of total lumbosacral flexion and true lumbar flexion measured by a dual inclinometer technique. Spine. 1994;19(23):2698-2701. PubMed ID: 7899966 doi:10.1097/00007632-199412000-00019

55. Karg M, Kulić D. Modeling movement primitives with hidden Markov models for robotic and biomedical applications. Methods Mol Biol. 2017;1552:199-213.

56. Sung PS. A kinematic analysis for shoulder and pelvis coordination during axial trunk rotation in subjects with and without recurrent low back pain. Gait Posture. 2014;40(4):493-498. PubMed ID: 25008865 doi:10.1016/j.gaitpost.2014.06.001

57. Hodges PW, Smeets RJ. Interaction between pain, movement, and physical activity: short-term benefits, long-term consequences, and targets for treatment. Clin J Pain. 2015;31(2):97-107. PubMed ID: 24709625 doi:10.1097/AJP.0000000000000098

58. O'sullivan P. It's time for change with the management of nonspecific chronic low back pain. Br J Sports Med. 2011;46(4): 224-227. doi:10.1136/bjsm.2010.081638

59. Marras WS, Wongsam PE. Flexibility and velocity of the normal and impaired lumbar spine. Arch Phys Med Rehabil. 1986;67(4): 213-217. PubMed ID: 2938557 
60. Crosbie J, Nascimento DP, Filho Rde F, Ferreira P. Do people with recurrent back pain constrain spinal motion during seated horizontal and downward reaching? Clin Biomech. 2013;28(8):866-872. doi: 10.1016/j.clinbiomech.2013.09.001

61. Pijnenburg M, Brumagne S, Caeyenberghs K, et al. Resting-state functional connectivity of the sensorimotor network in individuals with nonspecific low back pain and the association with the sit-tostand-to-sit task. Brain Connect. 2015;5(5):303-311. PubMed ID: 25557846 doi:10.1089/brain.2014.0309

62. Tsao H, Galea M, Hodges P. Reorganization of the motor cortex is associated with postural control deficits in recurrent low back pain. Brain. 2008;131(8):2161-2171. doi:10.1093/brain/awn154

63. Wand BM, Parkitny L, O'Connell NE, et al. Cortical changes in chronic low back pain: current state of the art and implications for clinical practice. Man Ther. 2011;16(1):15-20. PubMed ID: 20655796 doi:10.1016/j.math.2010.06.008

64. Claeys K, Dankaerts W, Janssens L, Brumagne S. Altered preparatory pelvic control during the sit-to-stance-to-sit movement in people with non-specific low back pain. J Electromyogr Kinesiol. 2012;22(6): 821-828. PubMed ID: 22595702 doi:10.1016/j.jelekin.2012.04.007

65. Pourahmadi MR, Ebrahimi Takamjani I, Jaberzadeh S, et al. The effect of core stabilization exercise on the kinematics and joint coordination of the lumbar spine and hip during sit-to-stand and stand-to-sit in patients with chronic nonspecific low back pain (COSCIOUS): study protocol for a randomized double-blind controlled trial. JMIR Res Protoc. 2017;6(6):e109. PubMed ID: 28572078 doi:10.2196/resprot.7378

66. Shum GL, Crosbie J, Lee RY. Energy transfer across the lumbosacral and lower-extremity joints in patients with low back pain during sitto-stand. Arch Phys Med Rehabil. 2009;90(1):127-135. PubMed ID: 19154839 doi:10.1016/j.apmr.2008.06.028

67. Kuo YL, Tully EA, Galea MP. Kinematics of sagittal spine and lower limb movement in healthy older adults during sit-to-stand from two seat heights. Spine. 2010;35(1):E1-E7. PubMed ID: 20042941 doi: 10.1097/BRS.0b013e3181c47a0e

68. Hirschfeld H, Thorsteinsdottir M, Olsson E. Coordinated ground forces exerted by buttocks and feet are adequately programmed for weight transfer during sit-to-stand. J Neurophysiol. 1999;82(6): 3021-3029. PubMed ID: 10601437 doi:10.1152/jn.1999.82.6.3021

69. Davids K, Glazier P, Araujo D, Bartlett R. Movement systems as dynamical systems: the functional role of variability and its implications for sports medicine. Sports Med. 2003;33(4):245-260. doi:10. 2165/00007256-200333040-00001

70. O'sullivan K, Clifford A, Hughes L. The reliability of the CODA motion analysis system for lumbar spine analysis: a pilot study. Physiother Pract Res. 2010;31(1):16-22. 